\title{
LA EFICACIA DEL SISTEMA EDUCATIVO EN ANDALUCIA
}

\author{
Manuela A. de PAZ BAÑEZ*
}

\section{INTRODUCCION}

A nadie le es ajeno la importancia que los aspectos sociales y económicos de la educación tienen en nuestro tiempo. Aún no compartiendo las ideas "desarrollistas" de los años sesenta, que consideraban a la educación como el principal factor del desarrollo económico y que, por tanto, un incremento de la oferta educativa (cualquiera que fuere) era considerado como la forma más eficaz de incrementar el crecimiento económico, si debe admitirse que la educación puede desempeñar un papel importante en el desarrollo económico y social, no sólo desde una perspectiva de incremento de la productividad, sino también como pieza clave dentro de una política de adaptación de la oferta, a la vez de medio que favorece la redistribución de la riqueza así como el bienestar y calidad de vida.

Todo esto justifica sobradamente la preocupación por los aspectos socio-económico de la educación. No obstante, en las dos últimas décadas se han producido unas circunstancias que intensifican esta preocupación. Ya en 1967 comienza a hablarse de la crisis de la educación ${ }^{1}$ provocada por el desfase entre los sistemas educativos y sus entornos sociales; agravado por la preocupación cuantitativa de incremento de las prestaciones educativas despreciando los aspecxtos cualitativos que estaban requiriendo profundos cambios para adaptarse a las nuevas exigencias de una sociedad en continua y rápida evolución. Estos desequilibrios, lejos de dismininuir, se han incrementando en los últimos años a pesar de los esfuerzos realizados por los gobiernos.

(*) Profesora de Estructura y Política Económica del Colegio Universitario de La Rábida. Universidad de Sevilla.

1. Ver P.H. COOMBS, La crisis mundial de la economía. Ed. Peninsular, Madrid, 1978 (1. ${ }^{a}$ Edición 1968). Y la reciente revisión sobre este tema, con el mismo título publicado por Ed. Santillana, 1985. 
En esta crisis los aspectos socio-económicos han jugado y juegan un papel fundamental. Durante muchos años, la escuela, la escolarización obligatoria y a ser posible gratuita, la prolongación de los años de escolarización e incluso la ampliación de las horas de permanencia de los alumnos en los centros, se han considerado como "un bien en sí" no suceptible de crítica, sólo cuando los gastos en educación han ido creciendo anualmente, en términos absolutos y relativos, en curvas ascendentes cuya proyección no indicaba ninguna propensión a la estabilización y mucho menos al descenso, sólo cuando la crisis económica ha golpeado las economías, se han planteado los gobiernos la necesidad de revisar sus gastos en educación. Esta preocupación ha sido precedida por la de los economistas y los expertos de las organizaciones internacionales quienes por primera vez formularon dos preguntas que a la mayoría de los educadores, más preocupados por la impartición de los saberes que por el costo de la enseñanza, les tomaron por sorpresa y parecieron impertinentes: ¿Se puede enseñar lo mismo a menos costo?, ¿Para qué sirve lo que se enseña?

La primera pregunta, en términos más técnicos, hace referencia al rendimiento o eficacia interna (aspecto relacionados con la calidad educativa) de los sistemas educativos, la segunda al rendimiento o eficacia externa o ajuste de la oferta escolar a la demanda social educativa, y en las dos, late la preocupación por los gastos en educación ${ }^{2}$.

Por último, para completar la argumentación sobre la importancia del tema podemos decir que se trata de una actividad (la educación) que absorbe el monto más elevado del presupuesto del estado y emplea a miles de personas; además de afectar directamente a más de la mitad de la población (entre alumnos, profesores y padres) e indirectamente a la totalidad. Sin entrar en aspectos más concretos como lo poco o nada que está estudiado este tema en nuestra región, la oportunidad de un estudio como éste ahora cuando las competencias en educación se han transferido a la Junta de Andalucía y la nueva etapa que con ello parece abrirse para nuestra región en este tema,...

Así, en este artículo, síntesis y primicia de los resultados de un amplio estudio desarrollado a lo largo de cinco años de trbajo y que pronto verá la luz ${ }^{3}$, trataremos, en primer lugar, los factores externos al sistema educativo que tiene una incidencia sobre él. En segundo lugar, hasta qué punto la infraestructura educativa actnal en nuestra región cubre las necesidades y demanda social. En un tercer momento expondremos algunas consideraciones sobre la calidad y rendimiento educativo; para terminar con las recomendaciones que consideramos mínimas para una planificación de la educación en Andalucía.

2. M.E.C. Elementos de Administración Educativa, Madrid, 1980.

3. Manuela A. de PAZ BAÑEZ, El sistema educativo en Andalucía. IDR. Universidad de Sevilla (en prensa). 


\section{CONDICIONANTES EXTERNOS DEL SISTEMA EDUCATIVO ANDALUZ}

Existe toda una serie de factores externos al sistema educativo que condicionan en gran medida sus resultados y eficacia. Estos factores son principalmente la misma estructura y organización del sistema y los factores físicos, demográficos, sociales, económicos y culturales del ámbito en el que se desenvuelve.

Estos factores influyen como grandes limitadores de las posibilidades del sistema educativo, y más en nuestra región donde sus niveles están muy por debajo de la media nacional.

En concreto, en cuanto al sistema educativo en sí, su estructura y organización, hay que decir que se encuentra muy condicionado por la historia y los problemas seculares mantenidos a lo largo de estos dos últimos siglos: dualismos y desequilibrios importantes como enseñanza pública-privada, general-profesional, media-universitaria, rural-urbana, ...; así como una oferta insuficiente y mal dotada, niveles de ineficacia y de fracaso muy acusados, falta de adecuación a la sociedad, enseñanza excesivamente elitista, ... No obstante, a lo largo principalmente de la década de los setenta la situación cambió en gran medida y la Constitución actual ha sabido poner las bases para la resolución de estos problemas, aunque se hace necesario un mayor esfuerzo para que esto sea una realidad.

Estos condicionamientos históricos del sistema educativo pesan decisivamente sobre la estructura y organización del sistema y dificultan su renovación y adecuación a la realidad actual en todos los aspectos.

En cuanto a la situación física hay que señalar el condicionante del relieve con grandes accidentes montañosos que agravan la situación de la mermada red de comunicaciones terrestres -intrarregionales y con el exterior-y el establecimiento de una red escolar equilibrada especialmente en las zonas de sierra.

En cuanto a la población, es de destacar un crecimiento demográfico mayor que a nivel nacional y, por tanto, la existencia de una población más jóven ( $29 \%$ de los andaluces tiene menos de 15 años frente al $25,6 \%$ a nivel nacional); es decir, más niños a escolarizar en proporción. También ha sido importante los movimientos migratorios al exterior e intraregionales (muy mitigados en los últimos años) que han incrementado los desequilibrios existentes en la red escolar (desertización de las zonas de sierra y aglomeración poblacional en la costa y áreas metropolitanas). Por último, un dato a favor de nuestra red educativa es el hecho de no encontrarse muy diseminada la población: sólo el 5,1 \% de los habitantes viven en pueblos de menos de 2.000 habitantes frente al $8,6 \%$ nacional. 
En cuanto a la situación socio-económica, es de sobra conocida su inferioridad en relación a otras zonas de España. La renta media no alcanza el 73 \% de la nacional y los niveles de paro son verdaderamente espectaculares (30,9\% del total de la población activa frente al $22,1 \%$ nacional ya de por sí muy elevado) a pesar de que Andalucía cuenta con una tasa de población activa por debajo a la nacional (43\% frente a $48 \%$ ). Los demás indicadores socio-económicos y de infraestructura básica coinciden en estas apreciaciones.

La densidad cultural ${ }^{4}$ y quizás sea el factor más determinante en el desarrollo y eficacia del sistema educativo y fruto a la vez de él, la situación es aún peor a la destacada para los otros factores educogénicos. Los índices de analfabetismo doblan la media nacional ( $11,8 \%$ frente a $6,6 \%$ en 1981), especialmente en las mujeres $(16,1 \%)$, en las zonas rurales $(15,3 \%)$ y en los habitantes de más edad (hasta los 20 años no hay diferencia con los niveles nacionales). El nivel de instrucción en general de la población es muy bajo (casi el $46 \%$ de la población no tiene estudios frente al $31 \%$ a nivel nacional). No obstante, parece que la situación está mejorando a lo largo de los años y la comparación de estos indicadores por edades es clara muestra de ello.

El resto de los indicadores de densidad cultural ( $n .^{\circ}$ de bibliotecas y nivel de lectura, cines, teatros, ediciones de libro,...) dan también valores muy por debajo de los nacionales y, por supuesto, de los deseables. Problema que no parece que esté en vías de solución dado la poca atención que a este tema se presta y los escasos recursos destinados a ello.

En resumen, podemos decir que en Andalucía los factores externos o educogénicos presentan una situación muy inferior a la media nacional (ya de por sí muy baja), cosa que influye de forma determinante en las posibilidades del sistema educativo, en su rendimiento y eficacia. De lo que se puede deducir la gran necesidad que tiene el sistem a educativo andaluz de recibir una atención especial para superar el bajo nivel educogénico con el que cuenta junto con los demás sectores de la economía que favorezcan el incremento de estos factores. No obstante, como veremos a continuación, esa atención especial no sólo no se ha producido, sino que la situación del sistema educativo también ha estado muy por debajo de los niveles alcanzados en otras zonas. Esto hace totalmente explicable el hecho de que en Andalucía se produzcan niveles de rendimiento muy inferiores a la media nacional.

4. Vid. M.A. de PAZ BAÑEZ, "La densidad cultural en Andalucía", Revista de Estudios Andaluces, n. ${ }^{\circ} 5$. 


\section{GRADO DE AJUSTE DEL SISTEMA EDUCATIVO A LA DEMANDA SOCIAL}

Pretendemos en este punto determinar la eficacia externa del sistema educativo a través de la confrantación entre la oferta educativa y la demanda social.

En cuanto a la oferta, sólo indicar que el $75 \%$ aproximadamente es pública con tendencia creciente en todos los niveles considerados excepto en Formación Profesional que sólo supone el $55 \%$. En la privada, la mayor parte $(81 \%)$ está subvencionada excepto en B.U.P.-C.O.U. que casi no existe subvención. Así, sólo el $5 \%$ de los puestos escolares en el conjunto de la enseñanza no universitaria está financiada por entes privados.

Su evolución en los últimos años ha sido estacionaria en E.G.B., de crecimiento moderado en B.U.P. y fuerte en E. Preescolar y F.P.; muy parecida a la evolución del número de alumnos. Se observa un fuerte incremento en la demanda de preescolar a pesar del descenso de la población en estas edades debido al escaso nivel de cobertura, y en F.P., fruto de un desplazamiento producido en los últimos años, de la demanda en las Enseñanzas Medias de B.U.P. (donde se encaminaban la mayor parte de los graduados) a F.P. donde hoy va una parte importante de estos graduados y los certificados que no abandonan el sistema (el $62 \%$ del total de los matrieulados de $1 .^{\circ}$ de F.P. son graduados); esto ha sido debido al aumento del prestigio de la F.P. aún muy poco considerada y la misma crisis económica que hace que las familias intenten que sus hijos comiencen a buscar trabajo pronto.

Esto ha provocado un estancamiento casi total en el número de alumnos en B.U.P. donde parece haberse llegado al punto de saturación y una subida espectaeular en F.P.

Por sexo, si en preescolar y E.G.B. no se producen diferencias importantes, en Enseñanzas Medias aún perdura una pequeña discriminación: abandonan más y antes las mujeres que los hombres; aparte de una particular preferencia de las mujeres por el B.U.P. y los hombres por la F.P.

Al comparar oferta y demanda se observa un claro desequilibrio en nuestra región que se refleja en la existencia de una demanda no satisfecha importante (hecho que se puede apreciar también en las bajas tasas de escolarización en relación a la media nacional); algo más de 150.000 niños en los niveles considerdos (10\% de la demanda global) especialmente en preescolar y enseñanzas medias. A la vez que sobran puestos escolares en otras zonas o en otros niveles en la misma zona. 
Las causas fundamentales de este hecho son: la falta de recursos que determina una falta de puestos escolares suficientes (especialmente en preescolar y F.P.), por un lado; y la ineficacia externa que provoca excesos y déficit de puestos escolares simultáneamente, por otra. Aparte de la existencia de una cierta reticencia de algunos sectores sociales para escolarizar a sus hijos.

Dado que el grado de cobertura en E. Preescolar y E.G.B. es - salvo excepciones-muy elevado, la existencia de demanda no satisfecha en estos niveles no parece sea debido a falta de puestos escolares en general aunque sí, en parte, a una falta de adecuación de estos puestos escolares a las necesidades concretas (sobran en E.G.B. y faltan en E. Preescolar por ejemplo). Por tanto, existen otras causas explicativas de estas demandas no satisfechas: desinterés de los padres, incapacidad física o psíquica de los alumnos, abandonos prematuros para trabajar -normalmente con los padres-, especialmente en las zonas rurales, lejanía del centro y necesidad de utilizar el transporte escolar durante bastante tiempo, el mismo fracaso escolar que hace abandonar prematuramente el centro,...

Pero donde se producen los desequilibrios mayores es en la Enseñanza Media, donde sobran en general puestos escolares en B.U.P. y faltan en F.P.; a parte del gran desequilibrio por comarca. No obstante, la elevada demanda no satisfecha en este nivel ( $27 \%$ del total) no es debido sólo a la falta de puestos escolares o a la mala distribución de los mismos; también se produce, como en la E.G.B., por otras causas, dado que el índice de utilización es bajo.

Así, podemos decir, que si bien en E.G.B. la organización de la oferta es bastante correcta y cubre en general las necesidades aunque deberían reconvertirse parte de los puestos escolares sobrantes en puestos de preescolar necesarios, y sólo existen algunos desequilibrios aislados; en las enseñanzas medias la organización racional de la oferta está lejos de conseguirse. Es necesario tanto un incremento en el número de puestos escolares ofrecidos en las zonas menos atendidas, como una reconversión de puestos escolares sobrantes de B.U.P.-C.O.U. en puestos escolares necesarios en F.P. Quizás la reforma de las enseñanzas medias solvente en parte este problema a través de la unificación en un "tronco común" hasta los 16 años y la articulación conjunta de las cinco especialidades que en principio se preveen para el $2 .^{\circ}$ ciclo (16-18 años).

También hay que hacer referencia a la diferente situación y problemática que se produce en las zonas rurales y urbanas. En las primeras, con una población en descenso, la tendencia es a que sobren puestos escolares en E. Preescolar y E.G.B.; aunque en las Enseñanzas Medias, dado que la atención a estas zonas siempre ha sido menor, el déficit es muy elevado. También provocada por la falta de interés de los alumnos para seguir estudiando una vez concluida la enseñanza obligatoria (índice de utilización muy bajo). Realmente el problema de la falta de voluntad de adquirir educación es quizás más grande, especialmente en estas zonas, que la escasez de oferta. 
En las zonas urbanas, por el contrario, con crecimientos poblacionales continuos y una población que demanda más educación en general que en las zonaś rurales, el problema es de déficit importante de puestos escolares especialmente en E. Preescolar y Enseñanzas Medias con índices de utilización elevados.

\section{ALGUNAS CONSIDERACIONES SOBRE CALIDAD Y RENDIMIENTO}

A pesar de todo lo dicho anteriormente, el problema de la falta de eficacia interna en Andalucía es sin duda muy superior al de la eficacia externa. Además su resolución puede resolver en gran medida las deficiencias en esta última.

Por esta razón, es necesario prestarle una atención más especial en su diagnóstico y posteriormente en su planificación. No obstante, es también la faceta menos estudiada del sistema educativo. Su dimensión y alcance así como sus causas y carencias se encuentran normalmente analizados de forma muy general, a partir de tópicos repetidos por todos y no muy contrastados empíricamente.

El rendimiento educativo es ciertamente muy bajo en Andalucía, aún más bajo que la media nacional. Las tasas de retrasos y abandonos son verdaderamente importantes. Las causas, aparte de lo referido más arriba de los factores externos e individuales, puede atribuirse al sistema educativo y su escasa eficacia interna; al que podemos considerar factor fundamental del bajo nivel de rendimiento que provoca —o no es capaz de superar - desigualdades entre los alumnos, aunque los factores individuales y sobre todo sociales sean un obstáculo importante en muchas ocasiones.

Dentro del sistema, los factores determinantes son el factor trabajo (falta de motivación, especialización, preparación,...) y el organizativo (empresa preindustrial, falta de flexibilidad, falta de autonomía de los centros, falta de profesionalidad de los administradores,...); sin contar la escasa adaptación (por exceso de inercia) del sistema educativo al sistema social y sus necesidades.

De hecho, la calidad de nuestro sistema educativo está muy lejos de ser la adecuada: el $20 \%$ aproximadamente de las unidades escolares públicas deben ser sustituidas por ser provisionales o estar en mal estado. Del resto, sólo las construidas después de 1970 disponen de los espacios suficientes. La situación del mobiliario y material didáctico también es muy precaria. El número de alumnos por unidad, sin embargo, no ha sido muy elevado en general en los últimos años; a excepción de los centros privados subvencionados y en los de B.U.P.-C.O.U. en algunas zonas urbanas. En cuanto al personal se aprecia la necesidad de profesores de apoyo y, especialmente, la necesidad de una regulación adecuada de la formación y especialización de los docentes; además de otros problemas como exceso de rotación, irregularidades con las sustituciones, ,... 
Pero quizás el factor que más influye actualmente en la calidad de un centro y que con menos recursos económicos se podría mejorar es el organizativo; y ello dada la gran complejidad de la educación en la actualidad y la necesidad de una adecuada coordinación e impulso de todos los factores implicados. Y es precisamente este factor el que necesita una atención mayor en Andalucía, no sólo por su importancia sino también por sus graves carencias. En la administración externa, el exceso de burocracia, la falta de agilidad, el retraso en la ayuda y asignaciones en el nombramiento de profesores,...; así como la falta de participación, de una normativa adecuada en algunos temas,... En la interna, si bien se ha realizado un enorme esfuerzo, aún se puede apreciar carencias importantes: falta de participación de los padres, falta de rodaje en la organización participativa de los centros en general, ausencia de profesionales de la administración y organización,...

Hemos destacado un gran grupo de carencias en la calidad educativa en nuestra región; no obstante, ¿cómo influyen esas carencias en el resultado y rendimiento educativo? Se trata una vez medidos los factores anteriores, de evaluar sus efectos. Para determinarlo se hace necesario un importante impulso en la investigación educativa en este campo; para poder determinar, una vez estudiados los costes, sobre qué factor conviene actuar para conseguir los máximos resultados con los mínimos costes. Dado que el estudio de la relación factores de calidad-rendimiento no nos toca a nosotros estudiarlo, nos hemos centrado sólo en el estudio de los costes para determinar la influencia de una medida que incremente la calidad en el presupuesto de educación.

Así, del análisis de costes como un indicador del funcionamiento y eficacia interna del sistema podemos deducir las siguientes conclusiones:

Los costes de personal, que suponen el $86,5 \%$ de los costes corrientes, son muy similares a los nacionales. Su peso en el coste total hace que cualquier medida de incremento en este factor suponga una repercusión importante en los presupuestos. Parecido, pero con menor importancia, ocurre con los gastos de reposición y conservación. No ocurre lo mismo, sin embargo, con los gastos en mobiliario y material didáctico que sólo supone un $2 \%$ aproximadamente y los organizativos que quizás sea el factor donde se puede establecer más medidas sin influencia sustancial en los costes. Es decir, son los factores organizativos los que se muestran más propicios para incrementar la calidad y con ello el rendimiento educativo; tanto por su influencia positiva en ésta (aun que esto sea una afirmación que necesita ser estudiada más detenidamente), como por su bajo coste.

Un dato significativo puede servir de ejemplo para corrobar la afirmación de que el verdadero problema del sistema educativo en Andalucía es su ineficacia interna. El ahorro en gastos corrientes debido sólo a la recuperación o eliminación de los retrasos podría llegar hasta un $28 \%$ del total; además, por esta misma 
razón, quedarían libres unos 400.000 puestos escolares en Andalucía que disminuirían casi a la mitad las necesidades de construcción. Sin considerar la eliminación del alto coste social que hay que pagar por este aspecto del proceso escolar y la eliminación en parte del retraimiento de la demanda por desánimo y abandono provocado por la falta de la calidad y el proceso escolar.

Además, el incremento de la calidad en los factores que provoca un coste mínimo y una rentabilidad (en cuanto a incremento del rendimiento escolar) máxima puede ser rentable incluso económicamente porque disminuiría el coste por alumno egresado por encima del incremento de coste por aumento de la calidad, aparte del decremento del coste social o beneficio social que podría producirse.

En todo esto nos falta un eslabón que es muy necesario estudiar en la actualidad y al que ya hemos hecho referencia: conocemos el incremento de coste que puede producir una mejora en algún factor de la calidad; conocemos también la reducción de coste que produciría el incremento en la eficacia interna del sistema; nos falta conocer el efecto en la eficacia que provocan las diferentes medidas de mejora de calidad, para determinar las medidas más adecuadas. Cosa que como ya hemos dicho debe ser fruto de la investigación pedagógica. Mientras tanto, aquí sólo podemos decir que según el coste, los primeros factores a considerar son los organizativos y de perfeccionamiento y motivación del profesorado, que a la vez parece puede dar resultados importantes en la eficacia; seguido de los materiales didácticos o herramientas tanto del profesorado como de los alumnos que pueden incrementar su productividad con poco esfuerzo. Por último, el factor que parece menos aconsejable por su elevada repercusión en el presupuesto educativo es el incremento en el número de profesores, aunque una orgauización profesional profunda si sería muy aconsejable.

\section{RECOMENDACIONES PARA UNA PLANIFICACION DE LA EDUCACION EN ANDALUCIA}

Todo lo dicho anteriormente nos lleva a proponer una serie de medidas para conseguir unos objetivos mínimos (aparte de subsanar las carencias determinadas). Las hemos agrupado en dos programas: Programa de creación y reconversión de puestos escolares y Programa de Ampliación y Mejoras; aparte del programa específico para la igualdad de oportunidades de cada zona: Programa de Educación Compensatoria.

Según el 1. ${ }^{\text {er }}$ programa, será necesario cubrir para 1991 un total de 280.000 puestos escolares (195.000 en E. Preescolar y E.G.B. y 83.000 en Enseñanza Media) para cubrir la demanda, de los cuales un máximo de $80.000(21,6 \%)$ podrían 
provenir de reconversiones entre bloques en una misma zona (aparte de la reconversión interna necesaria), además de 1.161 unidades más para reposición. Las necesidades se centrarán en las áreas urbanas mientras sobrarán en general puestos escolares en las zonas de sierra especialmente en E.G.B. y preescolar.

Las necesidades totales uniendo a lo anterior las debidas a reposición y amortización para Andalucía en unidades a construir o reconvertir ascienden a unas 21.455 (6.173 de nueva oferta y 15.288 para sustituciones) en E. Preescolar y E.G.B. y 5.657 (2.070 de nueva oferta y 3.587 para sustituciones) en Enseñanza Media. De ellas sólo 2.371 unidades pueden provenir de reconversiones de un nivel a otro dentro de la misma comarca; es decir, tendrán que ser construidas un total de 24.737 unidades (19.698 en preescolar y E.G.B. y 5.039 en medias).

Por tanto, tendrá que incrementarse el ritmo de construcciones experimentado hasta ahora, en un 15,5\% (inferior al incremento entre 1984 y 1985) y mantenerse en ese monto todo el período de planificación.

Esto parece conseguible. Aquí la dificultad superior no está en conseguir los puestos escolares necesarios sino en motivar a los alumnos para acudir al centro o para que no abandonen prematuramente; objetivo que sólo podremos lograr con medidas que incrementen la calidad de la educación y el atractivo de la escuela, unido a medidas compensatorias que eviten los problemas de desigualdad (geográfica y social) que se producen en nuestra región (programas de educación compensatoria concretos para cada comarca).

El Programa de Ampliación y Mejoras será, no obstante, más difícil de cubrir pero a la vez más necesario. En los centros privados las medidas a implantar serán el ajuste de las dotaciones de subvención a los verdaderos costes según una calidad mínima deseable; y el control y asesoramiento de dichos centros.

En los públicos, será necesario un gran número de medidas que se recogen en el punto anterior y que no parece necesario volver a enumerar aquí. Sólo decir que todas estas medidas de aumento de la calidad deberán ir encaminadas especialmente al incremento de la eficacia e igualdad de oportunidades. La primera con objeto de decrementar el número de repetidores y, por tanto, decremento de los costes tanto económicos como sociales; el segundo, incluido en los programas de educación compensatoria antes comentados, deberán favorecer la permanencia en el centro, la adecuación al ambiente concreto y el decremento del fracaso escolar. Deberá implantarse de forma gradual a lo largo del período de planificación, comenzando, como ya hemos dicho, por los que suponen un menor coste y mayores resultados tanto en eficacia como en calidad.

Por último, también deberá crearse grupos de experiencia y de investigación que analicen y aporten soluciones válidas a los problemas educativos concretos de nuestra región. Así como se fomentará el apoyo a las reformas que se consideran adecuadas a través de una mayor información y participación de todos los sujetos implicados. 
Sin embargo, todas estas medidas no resolverán el problema de la educación a $L / P$, dado que la reforma necesaria del sistema educativo es mucho más profunda (y especialmente de tinte organizativo). Por ejemplo, la reorganización de la profesión docente a través de una división racional del trabajo (enseñanza en equipo); así como la aplicación de una tecnología más acorde con las necesidades, que incremente la productividad de todos los factores. Todo esto incluido en una política de racionalización de la industria educativa que haga salir a este sistema de la situación preindustrial en la que se encuentra y que adecúe su realidad a las verdaderas necesidades y demandas de la sociedad.

En resumen, se hace necesario, una vez conseguido cubrir las necesidades de puestos escolares a $\mathrm{C} / \mathrm{P}$, comenzar toda una serie de medidas encaminadas a incrementar la eficacia sobre todo interna pero también externa (adecuación al medio y a la demanda) para que se consiga solventar las enormes carencias actuales; especialmente encaminado a que la empresa educativa consiga unos niveles adecuados en la sociedad posindustrial a la que debe dar respuesta.

Por último, decir que nos encontramos con un problema adicional constante en la planificación educativa al igual que en otras planificaciones: la limitación económica, que en definitiva no será decisiva si contamos con las financiaciones que se disponen hasta el momento; especialmente con la aportación del F.C.I. quizás incrementado en aproximadamente un 15 ó $20 \%$; aparte de las asignaciones del M.E.C. y los recursos propios y aplicación de fondos del F.E.D.E.R. Donde sí puede haber problemas es en la financiación de las medidas de mejoras, que necesitarán un monto quizás mayor al de construcciones; no obstante, si se realiza una buena jerarquización de objetivos puede irse consiguiendo todo lo pretendido. 